\title{
The Washington Double Star Catalog (WDS, 1996.0)
}

\author{
C.E. Worley and G.G. Douglass \\ U.S. Naval Observatory, 3450 Massachusetts Ave., N.W., Washington, DC 20392-5420, U.S.A. \\ e-mail: eqb@draco.usno.navy.mil
}

Received January 14; accepted January 23, 1997

\begin{abstract}
A major revision of the Washington Double Star (WDS) catalog containing data for the components of $78 \quad 100$ systems is now available from the data centers and via the World Wide Web (http://aries.usno.navy.mil/ad/wds/).
\end{abstract}

Key words: catalogs — binaries: visual

\section{WDS, 1996.0}

The Washington Double Star Catalog (WDS) maintained at the U.S. Naval Observatory by C.E. Worley and G.G. Douglass is the principal database of astrometric double and multiple star data for the astronomical community. The WDS Catalog contains positions (J2000), discoverer designations, epochs, position angles, separations, magnitudes, spectral types, proper motions, and, when available, Durchmusterung numbers and notes for the components of 78100 systems based on 451546 means. A new release is now available, WDS (1996.0), and is a major revision of the 1984.0 version. The stars with Durchmusterung numbers have been individually compared with the SIMBAD database. More precise magnitudes (photographic and photoelectric), and MK spectral types have been included where available. The WDS Notes have been extensively revised to include information on orbital motion and multiplicity (including astrometric, photometric, and spectroscopic), variability, composite spectra, etc. For pairs with orbits, the period, semi-major axis, and direction of motion are given. All stars with Durchmusterung numbers have also been matched with the major astrometric catalogs (ACRS, PPM, IRS, and FK5) in order to provide more accurate positions and proper motions. Since new data are continually being added to the database, the WDS is updated on a regular basis. Upon request, and within our resources, data from the Observation Catalog (the data base of $\approx 452000$ means comprising the WDS) for individual objects are supplied to professional and amateur astronomers world-wide (e-mail to eqb@draco.usno.navy.mil).

The WDS is available from the data centers:

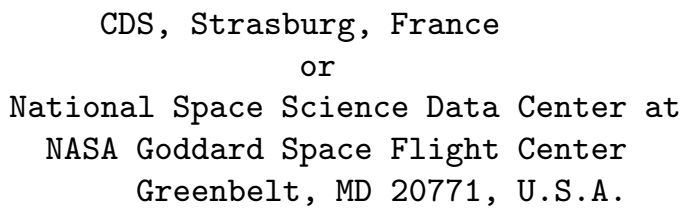

The WDS is also available on the World Wide Web at:

http://aries. usno.navy.mil/ad/wds/.

Acknowledgements. This research has made use of the Simbad database, operated at CDS, Strasbourg, France. 